\title{
Imaging findings in Erdheim-Chester disease: what every radiologist needs to know
}

\author{
Pawan Kumar ${ }^{1 A, B, C, D, E, F}$, Anuradha Sing ${ }^{1 A, B, C, D, E, F,}$, Shivanand Gamanagatti ${ }^{1 A, C, E, F}$, Sanjeev Kumar ${ }^{2 A, C, E, F}$, \\ Sheragaru Hanumanthappa Chandrashekhara ${ }^{3 A, B, D, E, F}$
}

'Department of Radiodiagnosis, All India Institute of Medical Sciences (AlIMS), Ansari Nagar, New Delhi, India 2Department of Cardiac Radiology, All India Institute of Medical Sciences (AllMS), Ansari Nagar, New Delhi, India

${ }^{3}$ Department of Radiology, IRCH, All India Institute of Medical Sciences, New Delhi, India

\section{Abstract}

\begin{abstract}
Erdheim-Chester disease (ECD) is a rare sporadic non-Langerhans cell histiocytic (LCH) proliferative disorder with systemic predilection. It usually affects adults in the $5^{\text {th }}-7^{\text {th }}$ decades of life and has non-specific clinical manifestations. Its suspicion is often heralded by the presence of characteristic radiological findings and subsequently confirmed by demonstration of CD68-positive xanthogranulomatous infiltrates on histopathology. Despite being a non-malignant entity, it might be fatal due to organ dysfunction. Imaging plays a key role in the diagnosis, management, and follow-up. Imaging findings are essential to establish the diagnosis, assess actual disease burden, and explore the aetiopathogenesis and therapeutic options to halt disease progression and associated morbidity.
\end{abstract}

Key words: diabetes insipidus, neurogenic, Erdheim-Chester disease, non-Langerhans cell histiocytosis.

\section{Introduction}

Erdheim-Chester disease (ECD) is an uncommon nonLangerhans cell histiocytic (LCH) proliferative disorder with multisystem involvement. Its peak incidence is in the $5^{\text {th }}-7^{\text {th }}$ decades of life with a slight male predilection, although children may occasionally be affected as well. Due to the rarity of the condition, its exact aetiopathogenesis remains to be established, and no definite genetic or infectious basis has been found yet. ECD is postulated to be an immune-mediated phenomenon resulting from an exaggerated proliferation of helper T cells, leading to the release of pro-inflammatory cytokines like interferons that results in the recruitment and activation of mast cells at the site of involvement [1-3].

Symptomatology of ECD is non-specific and depends on the site of involvement. Constitutional symptoms like fever, weight loss, night sweats are common, which may be mistaken for tuberculosis in the developing countries. Overall, skeletal involvement is the commonest, leading to bone pain as the presenting manifestation in the majority of cases. Most common extraosseous manifestations are central diabetes insipidus (DI) and exophthalmos; accordingly, a classical clinical triad of ECD includes bone pain, central DI, and bilateral exophthalmos.

Clinical, radiological, and light microscopic findings may be indistinguishable from LCH. Histopathologically, ECD is characterized by tissue infiltration by lipid-laden histiocytes. Demonstration of CD68 positivity in such xanthogranulomatous histiocytic infiltrates with simultaneous lack of CD1a and Birbeck granules is confirmatory, thereby aiding in differentiating ECD from LCH.

Like LCH, the sites and extent of extraosseous involvement determine the prognosis in ECD. Although involve-

Correspondence address:

S.H. Chandrashekhara, Department of Radiology, IRCH, All India Institute of Medical Sciences, Ansari Nagar, New Delhi, 110029, India,

e-mail: drchandruradioaiims@gmail.com

Authors' contribution:

A Study design · B Data collection · C Statistical analysis · D Data interpretation - E Manuscript preparation · F Literature search · G Funds collection 
ment of any organ system will result in its dysfunction, involvement of particular sites like central nervous system (CNS) and cardio-vascular system (CVS) certainly affects the prognosis due to the observed poor response to chemotherapy. Therapeutic advances have definitely lowered the morbidity, but the impact on mortality still remains dismal with reported 1- and 5-year overall survival of $96 \%$ and $68 \%$, respectively $[1,3-6]$.

\section{Imaging modalities}

An array of imaging modalities like radiography, ultrasonography (USG), computed tomography (CT), magnetic resonance imaging (MRI), and nuclear imaging may be performed depending on the site of involvement. Because ECD is a systemic disease with both osseous and extraosseous manifestations, imaging is used to ascertain the sites of involvement, delineate the extent, and monitor evolution during follow-up.

Plain radiography and Tc99 bone scintigraphy are useful in determining skeletal involvement. For the evaluation of thoraco-abdominal and CNS involvement, cross-sectional imaging techniques like CT or MRI are very useful. Due to its superior contrast resolution, MRI is preferred over $\mathrm{CT}$ in assessing CVS and CNS involvement. FDG-PET/ $\mathrm{CT}$ is invaluable for in vivo determination of disease activity during follow-up. Imaging findings along with clinical and demographic characteristics help to narrow the differential diagnosis and accordingly guide the pathology and immunohistochemistry for confirmation $[1,2,5,6]$.
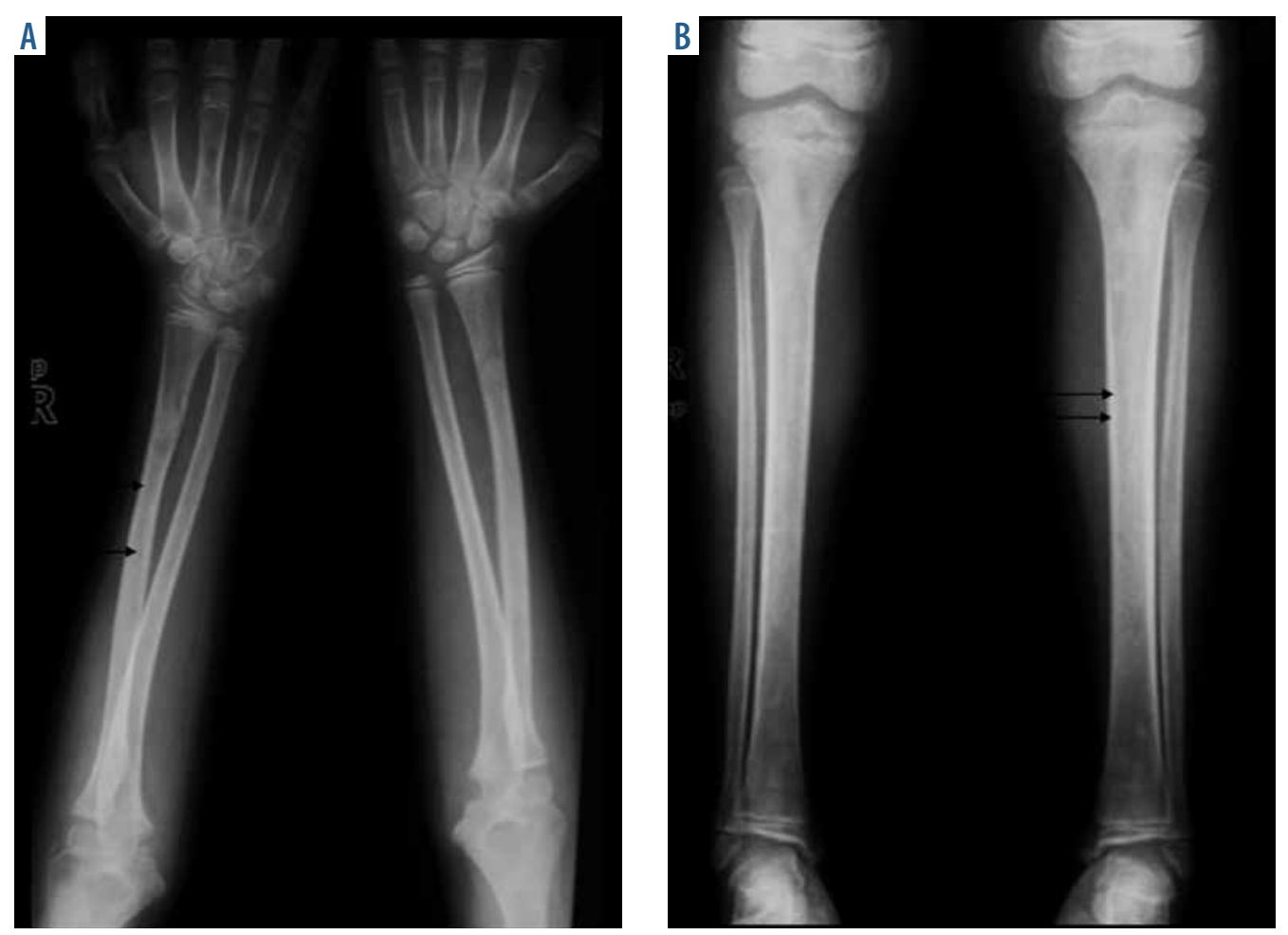

Figure 1. Anterior-posterior (AP) radiographs of both forearms (A) and legs (B) showing symmetrical osteosclerosis of the metaphyses and diaphyses of the long bones (black arrows) 


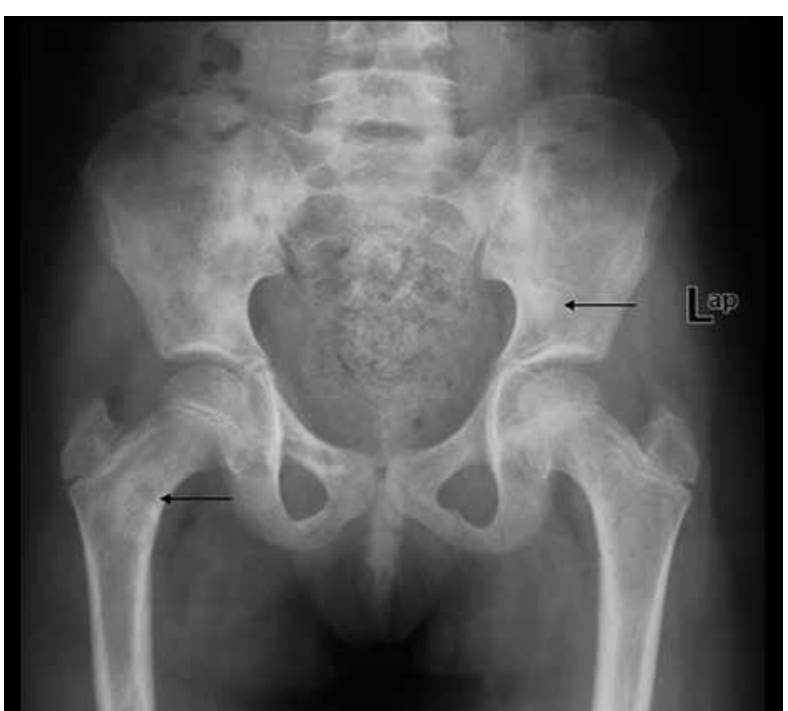

Figure 2. Anterior-posterior radiograph of the pelvis showing patchy osteosclerosis involving the bony pelvis and proximal right femoral shaft (black arrows)

findings; however, due to its better spatial resolution than Tc99m scintigraphy, it provides an added advantage of extraskeletal evaluation in a single scan.

In patients with osseous lesions, MRI is indicated for the assessment of extent of medullary involvement and to rule out associated osteonecrosis, which may occur primarily due to the disease or as an adverse effect of chemotherapy. Bone involvement on MRI is usually hypointense on T1WI and heterogeneously hyperintense on T2WI/ STIR images and shows enhancement $[4,5]$.

Differential diagnoses include other osteosclerotic conditions like lymphoma, chronic osteomyelitis, Paget's disease, or metastases; however, amongst these, symmetrical metadiaphyseal uptake on nuclear scintigraphy is exclusive to ECD. In contrast, there may be symmetrical radiotracer uptake in certain storage disorders like Niemann-Pick and Gaucher's disease, but bone lesions in these conditions are lytic [4-7].

\section{Central nervous system}

CNS involvement is seen in nearly $51 \%$ of cases and leads to death in nearly one-third of the affected individuals. The lesions are usually extra-axial, in contiguity with the facial or orbital bones, and may infiltrate the adjacent brain parenchyma. Clinical manifestations in CNS involvement are paralleled by the nature and location of the lesions. Osteosclerosis of calvarial and facial bones is a frequent concomitant finding in patients with CNS and maxillofacial involvement $[2,3,8,9]$.

\section{Extra-axial}

Overall, the commonest neurological manifestation in ECD is central DI resulting from the involvement of pos- terior lobe of the pituitary gland or infundibulum, which occurs relatively early in the course of disease. Further, diffuse involvement of the pituitary gland may result in hypopituitarism. MRI findings depend upon the extent of involvement and may vary from the absence of posterior pituitary bright spot on T1WI to the presence of enhancing nodules or micronodules in the infundibulum and hypothalamus.

Dural and meningeal involvement may be either diffusely infiltrating, or there may be formation of discrete masses. These lesions are hypo- to isointense on T1WI. Signal characteristics on T2WI are variable; however, T2 hypointensity, if present, is quite specific for ECD along with intense enhancement. Like vascular involvement in other conditions, there may be perivascular infiltration of the intracranial arteries and veins, which may lead to stroke.

\section{Intra-axial}

Although rare, intra-axial involvement may be either in the form of focal altered signal intensity or space occupying lesions. Signal alteration in the form of T2-hyperintensity has predilection for the posterior fossa and symmetrically involves the dentate and peridentate nucleus of the cerebellum without any enhancement. Occasionally, there may be involvement of the middle cerebellar peduncle. Owing to the lack of mass effect, these lesions tend to remain asymptomatic for long time; however, there may be cerebellar and pyramidal syndromes. Similarly, focal parenchymal deposits commonly involve the posterior fossa but may occur elsewhere as well.

Differential diagnosis: Neuroimaging findings in ECD may be indistinguishable from LCH. However, the pattern of skull bone involvement in LCH is usually lytic as compared to the osteosclerosis in ECD. Sarcoidosis and meningiomas may also have similar imaging findings. Nonetheless, concomitant orbital masses as well as osteosclerotic bone involvement is almost exclusive to ECD $[1,2,6,8,9]$.

\section{Orbit}

Orbital involvement is frequent in ECD, leading to exophthalmos, which is usually bilaterally symmetrical. Imaging is essential to localize the sites of involvement for deciding on further management, especially anatomical delineation prior to the surgery. CT shows infiltrating, hypodense soft tissue usually in retro-orbital, intraconal location, encasing the optic nerve and extraocular muscles (Figures 3 and 4). Orbital involvement is frequently multicompartmental with concomitant involvement of the extraconal, preseptal space, or the lacrimal gland. Although bone erosion is not a characteristic feature, osteosclerosis of the skull and facial bones may be present. On MRI, the soft tissue is isointense on both T1 and T2WI, and lacks enhancement $[3,6,10,11]$. 

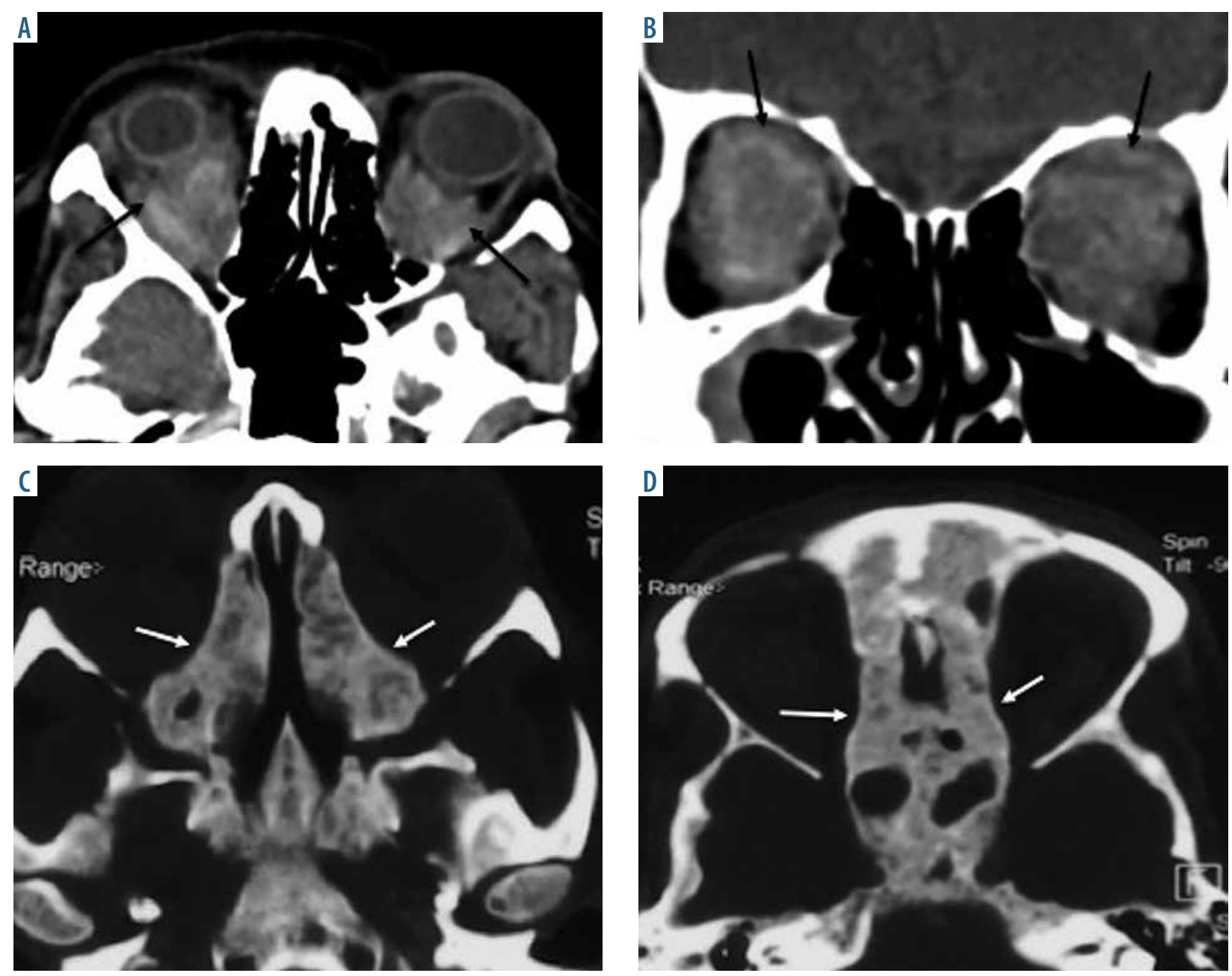

Figure 3. Bilateral proptosis and slowly progressive loss of vision in a 45-year-old male. A) Axial and coronal contrast-enhanced computed tomography (CECT) shows infiltrating, heterogeneously enhancing soft tissue in the bilateral retro-orbital, intra- and extraconal retrobulbar spaces (black arrows). B) Coronal reformat at the retro-orbital level showing its complete filling with indistinct extraocular muscles and optic nerves. C, D) Bone window shows osteosclerosis (white arrows) of the anterior cranial fossa skull base

Differential diagnoses for bilateral exophthalmos with multicompartmental involvement include Grave's disease, haematological malignancies (leukaemia, lymphoma), and sarcoidosis. In Grave's disease, extraocular muscle involvement is the main feature, leading to an increase in the muscle volume with sparing of the tendinous insertion. Peripheral blood smear and bone marrow examination aid in diagnosing haematological malignancies, whereas sarcoidosis may be distinguished based on the involvement of coats of eyeball and other systemic manifestations. With unilateral involvement, "orbital pseudotumour" is the leading differential diagnosis; however, extraocular muscles, including tendons, are primarily involved in psuedotumour [1-3,6,8,10,11].

\section{Pulmonary involvement}

Lung parenchyma and pleura are affected in about $40-50 \%$ of cases. Parenchymal involvement may be interstitial, alveolar, or combined. Patients tend to remain asymptomatic or may have progressive dyspnoea or cough. The most frequent finding on CT is smooth inter- and intralobular septal thickening; however, a lot of other non-specific findings like centrilobular nodules, cysts, consolidation, ground glass attenuation, or pleural effusion may also be seen. Septal thickening, due to histiocytic infiltration, has a lymphangitic distribution involving the peribronchovascular bundle, interlobular septa, and visceral pleura (Figure 5) [3,5,12].

Pleuroparenchymal involvement on imaging may be indistinguishable from other interstitial lung disease like LCH, sarcoidosis, pulmonary oedema, or venoocclusive disease. In LCH, classical cyst evolution from reticulonodular opacities with their bizarre shapes and apicobasal gradient favours the diagnosis. In contrast, in sarcoidosis, perilymphatic nodules, hilar and mediastinal lymphadenopathy are the main imaging findings. Septal thickening in cardiogenic pulmonary oedema is usually smooth, whereas it may be nodular in ECD. Accordingly, imaging findings may be correlated with clinical background and other systemic involvement to arrive at a definite diagnosis. Interstitial involvement in ECD 

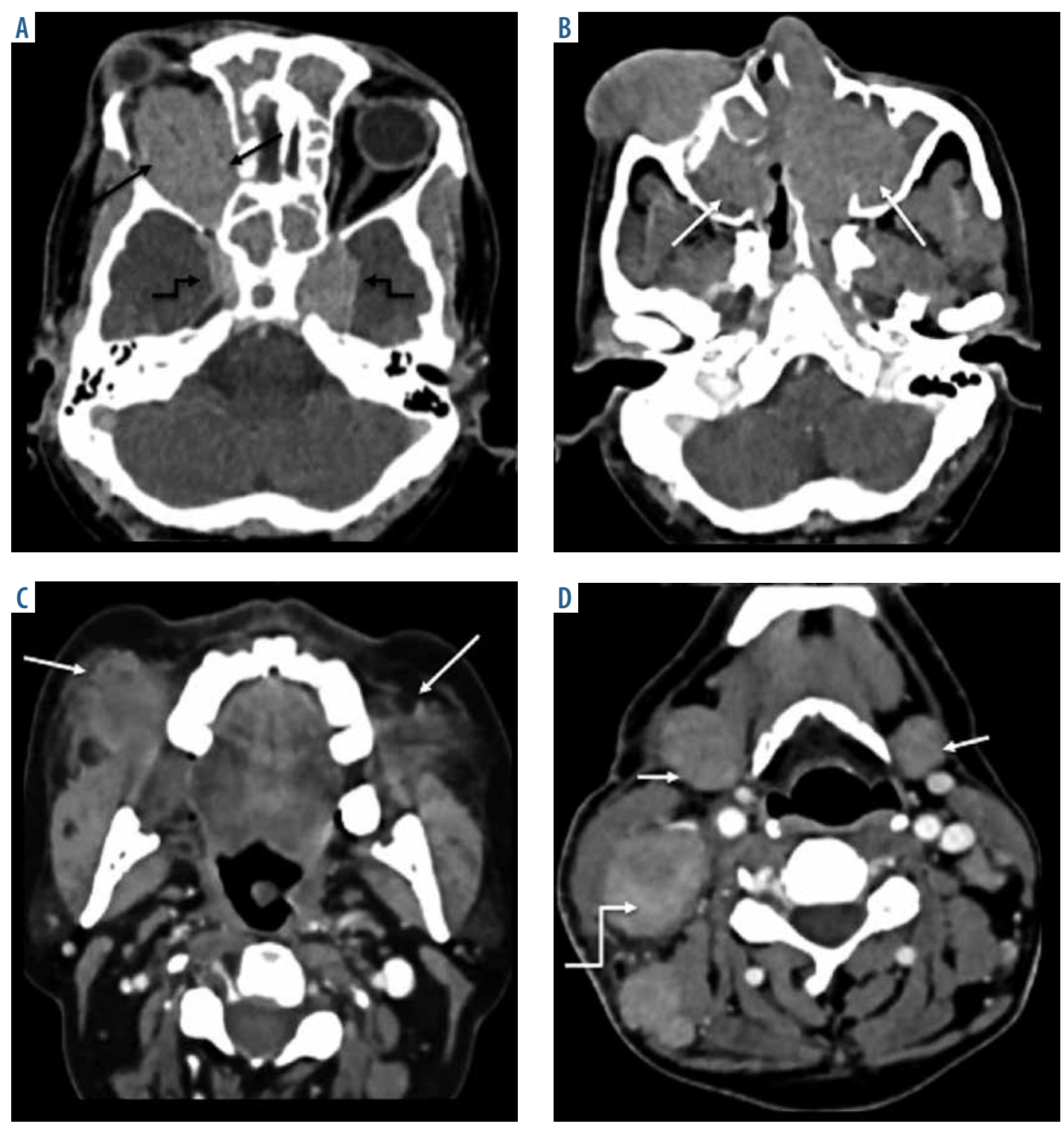

Figure 4. A 52-year-old male with axial proptosis in the right eye. Coronal contrast-enhanced computed tomography (CECT) shows (A) infiltrating, enhancing soft tissue in the right retro-orbital space (black arrows) encasing the extraocular muscles and optic nerve with contiguous extension through the orbital apex into the cavernous sinuses (left > right, curved black arrows). B) Similarly, attenuating soft tissue is also present in both sinonasal cavities (white arrows). C) Additionally, there was homogenously enhancing soft tissue in the bilateral masseteric space (right > left, white arrows) with (D) bulky submandibular glands and multicompartmental cervical lymphadenopathy (curved white arrow). No secondary bone changes are present

culminates in pulmonary fibrosis, leading to respiratory failure [1,3,5,12-15].

\section{Cardiovascular system}

Cardiovascular involvement is frequent in ECD and is seen in nearly three-fourths of cases. Similar to CNS lesions, cardiovascular system (CVS) involvement leads to a poor response to chemotherapy and leads to death in approximately $60 \%$ of cases, secondary to the compromise of either chamber filling or cardiac conduction defects resulting in myocardial infarction, arrhythmia, or valvular insufficiency. ECG findings are usually nonspecific, resembling any other cardiac conduction abnormalities. Although any part of the heart may be affected, the pericardium is most commonly involved (Figure 6). Accord- ingly, the disease process is postulated to stem from the subepicardial fat with subsequent myocardium involvement. Like elsewhere, there is soft tissue infiltration of the pericardium and/or myocardium. Pericardial involvement leads to its thickening and effusion. Myocardial infiltration more frequently involves the right heart, especially the right atrium and atrioventricular groove. On imaging, there is plaque-like, infiltrative, smooth thickening of the pericardium and/or myocardium that may involve the septum and valves $[3,5,15,16]$.

\section{Vasculature (aorta and its branches)}

The aorta and its branches are the leading sites of ECD involvement, which adversely affects the prognosis. It results in plaque-like, perivascular soft tissue encircling the aorta 

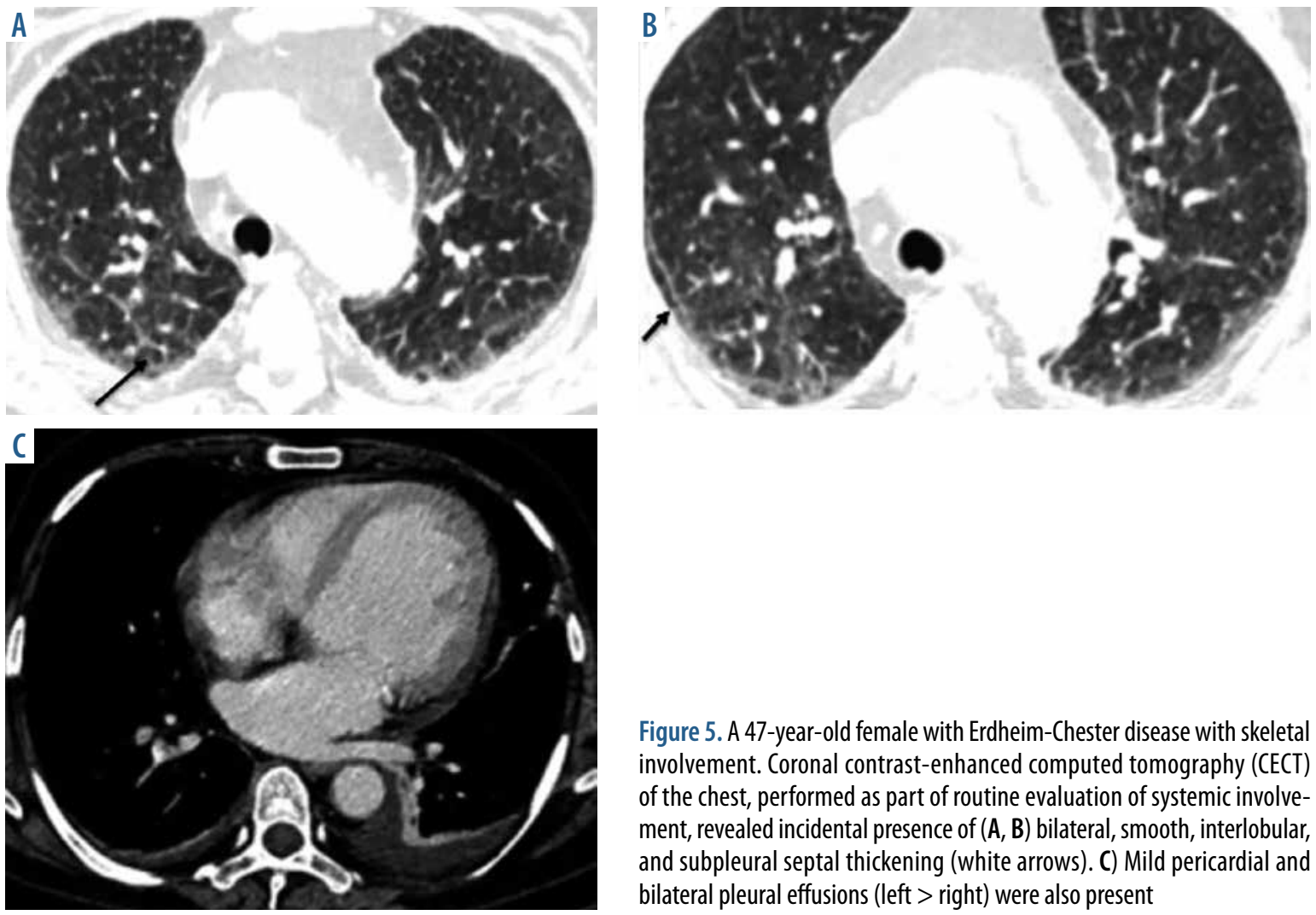

Figure 5. A 47-year-old female with Erdheim-Chester disease with skeletal involvement. Coronal contrast-enhanced computed tomography (CECT) of the chest, performed as part of routine evaluation of systemic involvement, revealed incidental presence of $(A, B)$ bilateral, smooth, interlobular, and subpleural septal thickening (white arrows). C) Mild pericardial and bilateral pleural effusions (left > right) were also present
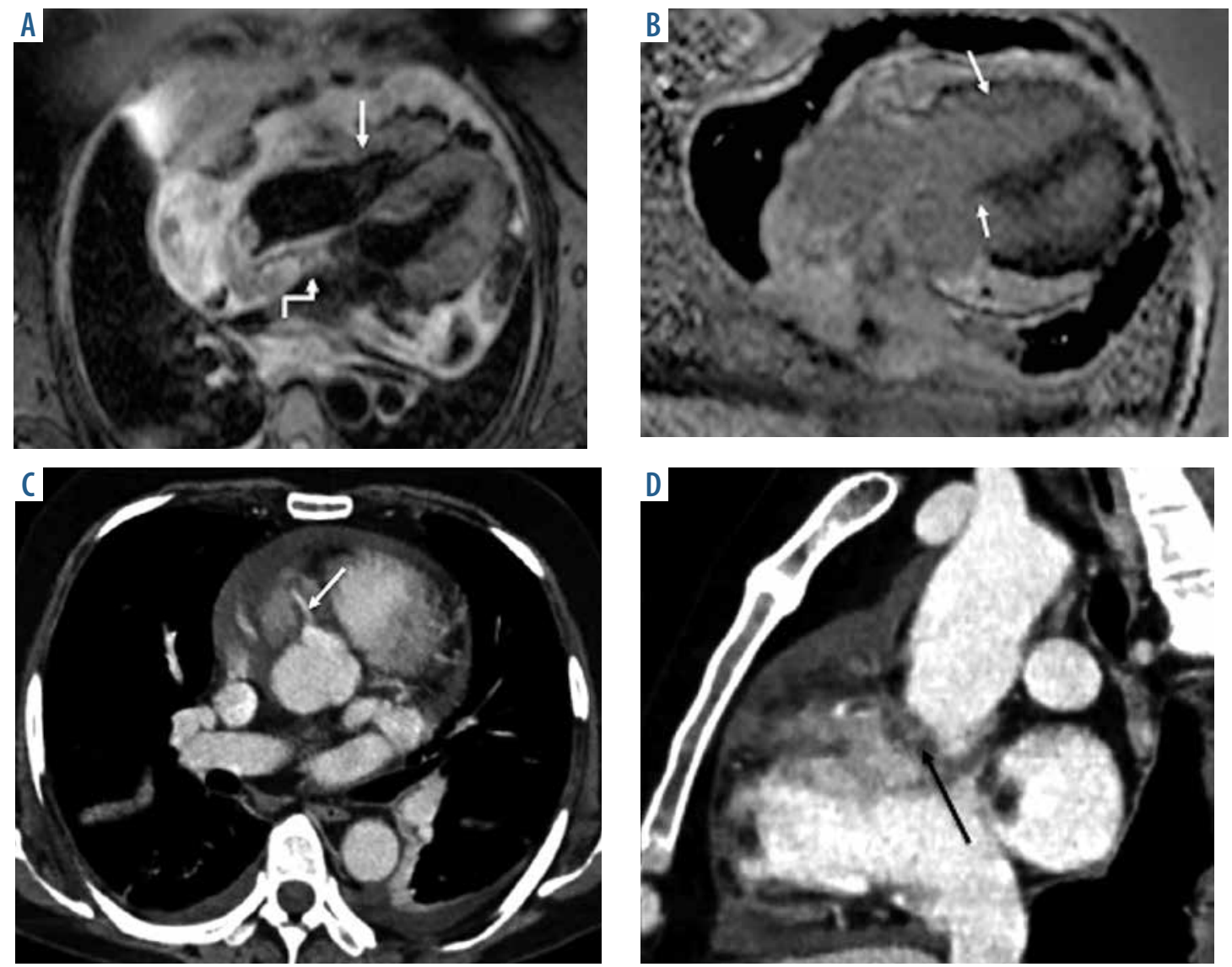

Figure 6. Cardiac magnetic resonance imaging of a 43-year-old female with dyspnoea and occasional syncopal episodes. A) Axial, ECG-gated, breath-hold, T2-weighted triple IR fast SE image showed mildly T2-hyperintense nodular, infiltrating deposits in the right ventricular free wall (white arrows) and the interatrial septum (curved white arrow). B) Axial, gadolinium-enhanced, ECG-gated, breath-hold, T1-weighted double IR fast SE image showed heterogeneous enhancement of the mural and septal thickening (white arrows). Note is made of moderate pericardial effusion and minimal right-sided pleural effusion. C) Coronal contrast-enhanced computed tomography (CECT) of the chest of a different patient with multisystem Erdheim-Chester disease involvement revealed enhancing soft tissue in the right atrio-ventricular groove encasing the coronary artery (white arrow) and (D) extending further to the root of the great vessels (black arrow) 
and its branches. Pathologically, the infiltration is adventitial rather than parietal. The pattern of involvement is variable and may be segmental with both symmetric and asymmetric circumferential forms. Perivascular soft tissue thickening may contiguously extend along the ostia of the branches of the thoracic and abdominal branches.

It may lead to extrinsic compression of the involved segment and consequent ischemic complications like cerebrovascular events, angina, or mesenteric ischemia depending on the site affected. Perivascular infiltration carries a poor prognosis as it ultimately resolves with fibrosis with persistence and progression of ischemic manifestations.

On CT, there is hypodense, plaque-like soft tissue circumferentially involving the aorta and its branches, also referred to as the "coated aorta sign". On MRI, it is isointense to muscle on both $\mathrm{T} 1$ and T2W images with subtle enhancement. MRI is advantageous over CT due to better assessment of vessel wall $[1,3,5,15,16]$.

Vascular involvement in ECD may be indistinguishable from other vasculitides, especially Takayasu arteritis. However, involvement of vessel wall is only peri-advential in ECD as compared to transmural in the latter condition. Hence, stenosis, thrombosis, aneurysms, and dilatation are more common in Takayasu arteritis. Retroperitoneal fibrosis is another leading site-specific differential diagnosis, which can be distinguished by lack of characteristic skeletal involvement as in ECD $[6,15,16]$.

\section{Kidneys and retroperitoneum}

Retroperitoneum including the kidneys is the most common extraosseous site to be involved, which is seen in ap-
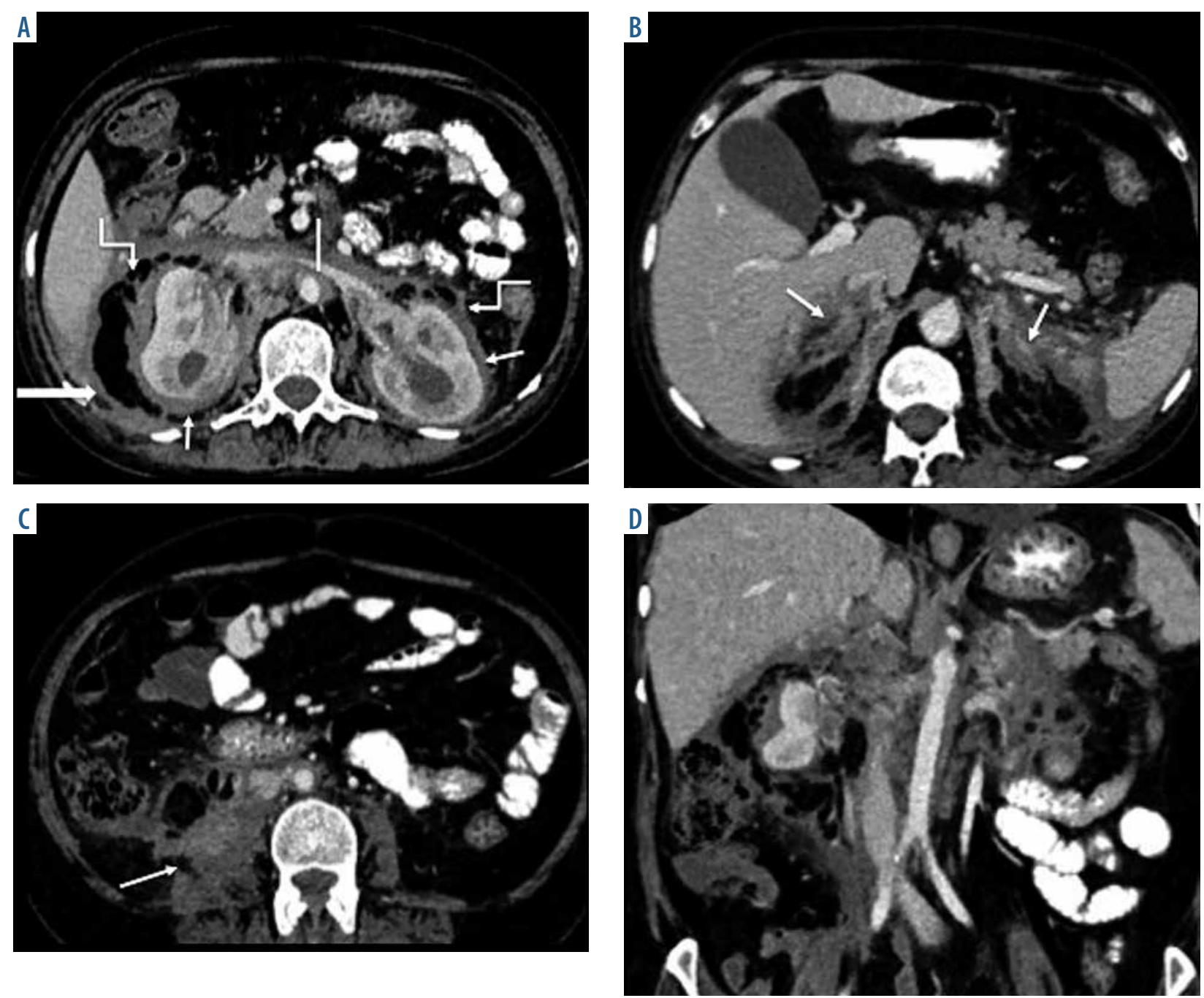

Figure 7. A 54-year-old female with abdominal discomfort and bone pain. Contrast-enhanced computed tomography (CECT) of the abdomen shows (A) ill-defined, plaque-like, hypodense soft tissue infiltrating the bilateral peri-renal spaces with thickened anterior and posterior renal fascia (white arrow). Of note are soft tissue strands extending laterally from the kidneys, i.e., the "hairy kidney sign" (curved white arrows), along with soft tissue in the region of renal pelvis causing mild hydronephrosis bilaterally. Also visible was further extension of this soft tissue in the anterior pararenal space, encasing the vessels including the aorta but characteristically sparing its posterior space, i.e., the "coated aorta sign" (white line). Laterally, there was bilateral thickening of lateroconal fascia with presence of perihepatic soft tissue along the right lobe of the liver (solid arrow). B) Both adrenal glands were also thickened and enhancing (white arrow), with surrounding fat stranding; however, there was no adrenal dysfunction. C) Caudally, there was infiltration of the right psoas muscle (white arrow). (D) Coronal image showing coated aorta along with perirenal and perihepatic soft tissue thickening 
Table 1. Summary of systemic involvement in Erdheim-Chester disease

\begin{tabular}{|c|c|}
\hline System & Radiological findings \\
\hline Skeletal system & $\begin{array}{l}\text { Bilateral patchy symmetrical cortical and medullary sclerosis with trabecular coarsening involving meta-diaphysis } \\
\text { of appendicular bones sparing the epiphysis. } \\
\text { Tc99 scintigraphy: symmetric radiotracer uptake at the end of long bones. } \\
\text { Ancillary findings: Bone infarcts. Uncommonly, partial epiphyseal involvement, periostitis. }\end{array}$ \\
\hline Central nervous system & $\begin{array}{l}\text { Extra-axial: More common. } \\
\text { Pituitary gland: Posterior lobe of pituitary, infundibulum. } \\
\text { Dural deposits, dural/pachymengeal thickening, perivascular thickening. } \\
\text { Intra-axial: Abnormal symmetrical } T 2 \text { hyperintensity with no contrast enhancement or mass effect involving the bilateral } \\
\text { dentate and peridentate nuclei. } \\
\text { Focal masses commonly in posterior fossa. } \\
\text { Osteosclerosis of the maxillofacial skeleton. }\end{array}$ \\
\hline Cardiovascular system & $\begin{array}{l}\text { Plaque like soft tissue in mediastinum. Infiltration of cardiac chambers (commonly right), pseudo-tumoral soft tissue } \\
\text { deposits in the atrioventricular sulcus. } \\
\text { Ancillary findings: pericardial thickening, effusion. }\end{array}$ \\
\hline Pulmonary & $\begin{array}{l}\text { Interlobular septal thickening, fissural thickening, centrilobular nodules, ground glass attenuation, consolidation, pleural effusion. } \\
\text { Interstitial pattern more common. }\end{array}$ \\
\hline Aorta and its branches & Periarortic soft tissue encasing aorta ("coated aorta") and its branches. \\
\hline Retroperitoneum & Soft tissue in perirenal and pararenal spaces "hairy kidney sign". \\
\hline Orbit & Retro-orbital intraconal soft tissue usually bilateral leading to exopht \\
\hline
\end{tabular}

proximately $68 \%$ of cases. As elsewhere, the majority of patients remain asymptomatic or may have non-specific clinical manifestations like abdominal pain or dysuria. In early stages, large, infiltrating, soft tissue masses are present in bilateral perirenal and posterior pararenal spaces, causing mass effect on the kidney. The retroperitoneal soft tissue deposit is hypoattenuating on CT (Figure 7). On MRI, it is isointense to muscle on both $\mathrm{T} 1$ and $\mathrm{T} 2 \mathrm{~W}$ images, with mild homogeneous enhancement. Perirenal fat infiltration results in spiculated appearance, termed the "hairy kidney sign". As the disease progresses, these soft tissue masses compress the pelvicalyceal system or ureters leading to obstructive uropathy. Additionally, there may be involvement of adrenal glands; however, despite bilateral involvement, adrenal insufficiency is rarely encountered [5].

The major differential diagnosis with such pattern of involvement is retroperitoneal fibrosis, either primary or secondary. In early stages, the pattern of involvement may aid in distinction as primary retroperitoneal fibrosis commences proximally from the aortic bifurcation, whereas ECD is epicentred at the level of renal hilum. Accordingly, distal ureters are most commonly affected in RPF as opposed to the renal pelvis and proximal ureter in ECD. Additionally, RPF does not involve the peri-renal space and in non-neoplastic causes of RPF there is sparing of the retro-aortic space, although compression of IVC may be present in both $[3-6,15,16]$.

\section{Unusual sites}

ECD may potentially involve any site including skin, breast, lymph nodes, thyroid gland, testis, and visceral organs. Skin involvement may be plaque-like or nodular, also known as xanthomas and xanthelasmas. Involvement of solid visceral organs and the mesentery is extremely rare $[1,6,15]$.

\section{Conclusions}

ECD is a rare, non-LCH histiocytic proliferative condition with systemic predilection that usually affects the elderly. Amongst the myriad of non- specific manifestations, precise identification of this entity is essentially a multidisciplinary approach based on clinical and demographic profile, radiological findings, and final confirmation on histopathology. Despite being a non-neoplastic condition, its prognosis is dismal and is largely dependent on the sites and extent of extraosseous involvement. CNS and CVS involvement confers a poor response to chemotherapy and an inexorable downhill course. Knowledge of its imaging findings (Table 1) is essential to avoid misdiagnosis, initiate treatment, and determine its exact incidence, natural course, and therapeutic guidelines which remain to be established due to its rarity.

\section{Conflict of interest}

The authors report no conflict of interest. 


\section{References}

1. Veyssier-Belot C, Cacoub P, Caparros-Lefebvre D, et al. ErdheimChester disease. Clinical and radiologic characteristics of 59 cases. Medicine 1996; 75: 157-169.

2. Mazor RD, Manevich-Mazor M, Shoenfeld Y. Erdheim-Chester disease: A comprehensive review of the literature. Orphanet J Rare Dis 2013; 8: 137.

3. Lodhi U, Sarmast U, Khan S, et al. Multisystem radiologic manifestations of Erdheim-Chester disease. Case Rep Radiol 2016; 2016 2670495.

4. Dion E, Graef C, Miquel A, et al. Bone involvement in Erdheim-Chester disease: Imaging findings including periostitis and partial epiphyseal involvement. Radiology 2006; 238: 632-639.

5. Antunes C, Graça B, Donato P. Thoracic, abdominal and musculoskeletal involvement in Erdheim-Chester disease: CT, MR and PET imaging findings. Insights Imaging 2014; 5: 473-482.

6. Munoz J, Janku F, Cohen PR, et al. Erdheim-Chester disease: Characteristics and management. Mayo Clin Proc 2014; 89: 985-996.

7. Kim MS, Kim CH, Choi SJ, et al. Erdheim-chester disease. Ann Dermatol 2010; 22: 439-443.

8. Drier A, Haroche J, Savatovsky J, et al. Cerebral, facial, and orbital involvement in Erdheim-Chester disease: CT and MR imaging findings. Radiology 2010; 255: 586-594.
9. Sedrak P, Ketonen L, Hou P, et al. Erdheim-Chester disease of the central nervous system: New manifestations of a rare disease. Am J Neuroradiol 2011; 32: 2126-2131.

10. De Abreu MR, Chung CB, Biswal S, et al. Erdheim-Chester disease: MR imaging, anatomic, and histopathologic correlation of orbital involvement. Am J Neuroradiol 2004; 25: 627-630.

11. Alper MG, Zimmerman LE, Piana FG. Orbital manifestation of Erdheim-Chester disease. Trans Am Ophthahalmol Soc 1983; 81: 64-85.

12. Rush WL, Andriko JA, Galateau-Salle F, et al. Pulmonary pathology of Erdheim-Chester disease. Mod Pathol 2000; 13: 747-754.

13. Pan A, Doyle T, Schlup M, et al. Unusual manifestation of ErdheimChester disease. BMC Gastroenterol 2011; 11: 77.

14. Wittenberg KH, Swensen SJ, Myers JL. Pulmonary involvement with Erdheim-Chester disease: Radiographic and CT findings. Am J Roentgenol 2000; 174: 1327-1331.

15. Dion E, Graef C, Haroche J, et al. Imaging of thoracoabdominal involvement in Erdheim-Chester disease. Am J Roentgenol 2004; 183: $1253-1260$.

16. Haroche J, Cluzel P, Toledano D, et al. Images in cardiovascular medicine. Cardiac involvement in Erdheim-Chester disease: Magnetic resonance and computed tomographic scan imaging in a monocentric series of 37 patients. Circulation 2009; 119: e597-598. 